\title{
WIRELESS SENSOR NETWORKS FOR FIRE DETECTION: A SURVEY
}

\author{
Snehal Bagade ${ }^{1}$ \\ ${ }^{I} P G$ student at SVERI's College of Engneering, Pandharpu
}

\begin{abstract}
This paper describes the use of the wireless sensor network for fire detection. There are many field areas such as industries, residential area, mines, forest etc. where fires take place. In such case if we early know the fire place then we can save the lives of workers or peoples in that area and also save the property. This paper gives survey of the use of WSN in such cases. WSN have the broad area of application, hence here I describe what WSN is and how it works.
\end{abstract}

Keywords: Wireless sensor network (WSN), fire

\section{INTRODUCTION}

Fire is one of the main disasters in human life; if it takes place then we cannot control it. There are lots of fire incidents taken place in day to day life. For longer than recorded history, fire has been a source of comfort and catastrophe for the human. Fire is rapid, self-sustaining oxidation process accompanied by the evolution of heat and light in varying intensities. Fire is believed to be based on three elements being present: fuel, heat and oxidizer. Fire disasters can occur above the ground (in tall buildings and on planes), on the ground, and below the ground (in mines). Sometimes they occur in circumstances that are unexpected or unpredictable.

In India an average, about 25,000 persons every year die due to fires and related causes. It is estimated that about 42 females and 21 males die every day in India due to fire. According to the statistics released by the National Crime Records Bureau, fire accounts for about $5.9 \%(23,281)$ of the total deaths reported due to natural and un-natural causes during the year 2012. According to one estimate the major losses reported by the Indian Insurance Companies in the year 2007-2008 indicate, that about $45 \%$ of the claims are due to fire losses. Another estimate states that about Rs. 1000 crores are lost every year due to fire. Fire losses are reported both in industrial and non-industrial premises like hospitals, commercial complexes, educational institutions, assembly halls, hotels, residential buildings, etc. According to Fire Risk Survey (FRS) 2013, carried out by Pinkerton \& Federation of Indian Chambers and Industry, in India, fire accounted for $8.45 \%$ of the overall ranking of risks. FRS also revealed that fires have been rated as the 5th highest risk in industry in 2013.

The survey point out that during the period 2009-2012, Mumbai fire department had attended 13,185 incidents of fire out of which 9711 were caused due to defective electric circuit.

\section{List of Major Work Place Fire Hazards}

Flammable chemicals: found in laboratories, shops, art studios, maintenance activities (painting, cleaning, auto repair...) engines, boilers and other heating appliances.

Processes involving open flame: Welding, brazing and similar operations, cooking, smoking, and some lab operations.

Heat producing devices: Drying (both in the laundries and laboratories), cooking, heat producing devices such as hot plates and space heaters

Use and disposal of chemicals: Experiments in labs, hazardous waste handling, oily rags in art studios, and shops.

Electrical equipment: Short circuits and malfunctioning equipment.

Hence early fire detection systems are important to avoid the losses of life's as well as property. The system based on WSN is one of the good systems which can provide early fire detection. The part 1 of the paper describe the WSN, part 2 describe the different application areas of WSN for fire detection and part 3 describe the proposed system for fire detection I am going to develop.

\section{PART 1: WIRELESS SENSOR NETWORK}

Wireless sensor networks are consists of the sensing device such as sensors, processing device such as microcontroller, and communicating device as transceiver. The sensors are low cost, small in size, and communicate in short range. The microcontrollers are used to execute task, data processing and assist the functionality of other components in the sensor node. The memory is used for data storage while the transceiver acts from the combination of transmitter and receiver functions and used for communication purpose. Due to all small size components they called as tiny nodes. Sensor nodes enable environment sensing together with data processing. Consists with a variety of sensors, such as 
temperature, humidity, light sensing, fire, smoke, volatile component detection, allow monitoring of different environmental parameters. Natural phenomena data such as temperature, light, sound, pressure etc. are collected by sensors and processed by the microcontroller as per requirement. Then this data is transmitted to a server. These battery powered nodes are used to monitor and control the physical environment from remote locations. Figure 1 shows the WSN architecture.

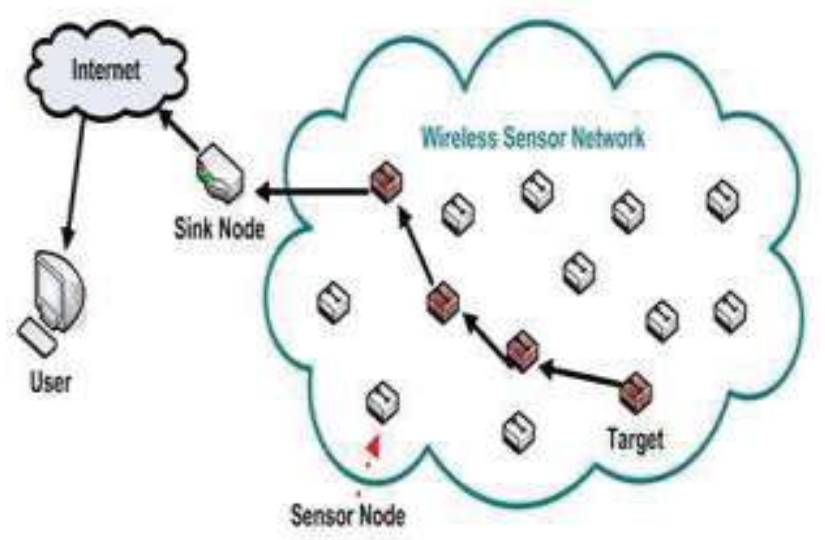

Fig 1

The Wireless Sensor Network architecture shown above, it contains sensor nodes, user and sink node. Sensor nodes will communicate with each other and transmit the processed data to sink node over a wireless communication. Sink node collects data from all the nodes, and transmits the analyzed data to user via Internet.

Recent advances in wireless communications and electronics have enabled the development of low-cost, lowpower, multifunctional sensor nodes that are small in size and communicate in short distances. These tiny and generally simple sensor nodes consist of sensing units, data processing, and communicating components.

\section{PART 2: APPLICATIONS}

There are different application areas where WSN is used for fire detection such as forest, mines, industries, residential area etc. In each case if fire taken place and if we can't get early detection then it becomes catastrophic. Hence WSN is the technology used.

\subsection{Industries}

There are different industries where fires take place such as chemical industries, cracker industry, sugar factory, automobile industries etc. Industrial fires and explosions have too much loss, cost companies and governments billions of rupees loss every year, and loss of life's, which can't be described in monetary terms. According to the most recent fire statistics from the National Fire Protection Association (NFPA): An average of 37,000 fires occurs at industrial and manufacturing properties every year. These incidents result in: 18 civilian deaths, 279 civilian injuries, and $\$ 1$ billion in direct property damage.

Most common reason for industrial fires is:

1. Combustible dust: This is the most deadly in different types of industries such as food manufacturing, woodworking, chemical manufacturing, metalworking, pharmaceuticals etc.

2. Hot work: Hot work is one of the causes of fire in industries.

3. Flammable liquid and gasses: This cause taken place in chemical industries

4. Equipment and machinery: Faulty equipment and machinery are also major causes of industrial fires.

5. Electrical hazards: Electrical fires are one of the top five causes of fires in manufacturing plants.

In [4] proposed the fire detection system for cracker industries having some requirements as energy efficient detection, earlier detection and precise location, with stand to harsh environment, Auspicate capacity. In [5] describe the use of the WSN in oil and gas industry with their requirements as nodes of WSN in industry gone from abrasive environments and also placed at the harsh places.

\subsection{Forest}

In how much time we notice that there is fire in forest? Most of the times, when someone notices about the fire, it is too late because the fire has spread. Fires naturally occur in nature but certain mitigating circumstances can cause the number of forest fires that break out to rise. In 2016 year in India the number of forest fires has increases by 55\%. This number was reported by the Parliamentary Standing Committee on Science and Technology in India on December 16. Several causes for the rapid spread of these fires include drought, hot weather, and accumulated Chir pine needles which are inflammable due to their high-resin content and as such provide an abundant source of fuel for these wildfires.

Forest is main part in human life, which provides life support to wild animal. If fire take place in forest then lot of damage take place. In [2] describes the hierarchical wireless sensor network which is used for early fire detection at risky area. This consists of the sensors nodes (SN) which collect data from the environment and central nodes gather data from SNs and transmit the information to the control centre. In [12] it is discuss that WSN is used for forest-fires surveillance system in South Korea Mountains. This system consists of WSNs, middleware and web application. The WSNs used to measure temperature, humidity and detect smoke. The middleware program and the web application analyze the collected data. This system detects the heat and gives the early alarm in real time when forest fires occur. 


\subsection{Mines}

In India over 65 percent of India's power supply is generated from coal. Mining of coal in Jharia started in 1894, and has increased ever since. Today, Jharia is home to two large underground and nine large open cast mines. But now days there are too many mines in Jharia are with the fire.

There are different mines all over the world such as coal mines, different metal mines, fossil fuels etc. The underground environment is not good enough and accidents happen frequently. In Fire is a major concern for those who work in underground coal mines. Coal mine fire can occur at any time and often results in partial or total evacuation of mine personnel and could result in the loss of lives. In [7] wireless sensor network is used for fire detection in bord and piller coal mines. The system tries to give the exact location of the fire and also give the prevention system to avoid the loss of lives and the natural resources. In [10] design the integrated mine network consists of WSN and other communication technologies such as fibers, leaky cables so on. In this WSN is used for monitor the long roadway, while fiber is used as backbone network. Hence cost of the system is reduced and use of the present system is also done.

\subsection{Residential Areas}

We know that there are different fire incident take place in residential areas accidently or due to short circuit or also carelessness of people. Fire incidents happen frequently in residential areas; hence if we have the system then we can control the accidents. Hospitals are also included in the fire concern area.

In [1] discuss the WSN based on location sensitivity information, as location of the fire is main concern. If we get the early fires exact location then we can control it at early stage. In [9] develop the fire monitoring system for home and hospitals by using the ZigBee. It uses three types of nodes such as monitoring nodes to monitor the presence of intruder and temperature of that area, intermediate node and extinguishing node used when there is an enormous increase in the temperature, the fire extinguishing unit to switch $\mathrm{ON}$ the extinguisher that avoids the impact of fire accidents. In [11] design the WSN for detecting the fire in high rise building. These detectors consist of temperature sensor of smoke sensors. All these detectors deployed in building in hierarchical manner all over the floor and detect the changes as per requirement.

\subsection{Environmental Monitoring}

Environmental monitoring is one of the important applications of WSN. The use of WSN for it grows widely along with the development of the technology. These environmental monitoring system monitors environment parameters such as temperature, humidity, light, pressure etc. In [8] discuss the different environment monitoring system application such as agriculture monitoring, Habitat Monitoring, Indoor living monitoring. In [6] discuss the WSN for the fire emergency and gas detection for both indoor and outdoor environments. In [3] explains the use of the WSN for poly house monitoring by using the LEACH protocol, to avoid the energy loss. Energy saving is also one of the main constrain for WSN. Hence they explain how the energy loss is avoided by using this protocol.

\section{PART 3}

The block diagram of the given system is shown in the below figure 2. It consists of different components such as microcontroller, different sensors such as temperature, light dependent register (LDR), fire, security, gas sensors and LCD display, GSM modem for communication and the different devices that are control by using microcontroller.

The microcontroller used is act as a processing unit of the sensor node system. All the sensors are interface with the microcontroller. The different physical quantities sensed by different sensors are sending to the microcontroller. These sensed values are compare with the threshold values and if these values are higher than the threshold value then this information is send to the base station i.e. control cabin. The different devices are control by through the microcontroller. The sensed data by the sensor is send through the GSM modem to the control cabin.

Different sensors are used for different purpose. The temperature sensor is used to monitor the temperature of the surrounding and with respect to change in the temperature the $\mathrm{dc}$ fan is activated by the controller, to keep temperature normal. The LDR are used to measure the light intensity present in the environment so as when it starts getting dark the controller switch on the lights. The gas sensor senses the presence of gas in the industry equipment's. Fire sensor detects the presence of fire in the atmosphere.

If this sensor is activated then the buzzer will be activated which will alarm the respective authority. The TSOP sensors are used which detects the presence of the person. The relays are used to control the different devices. If there is fire take place then the water sprinkler is active through the microcontroller. The LCD display is used to display the different values of the physical quantities. 

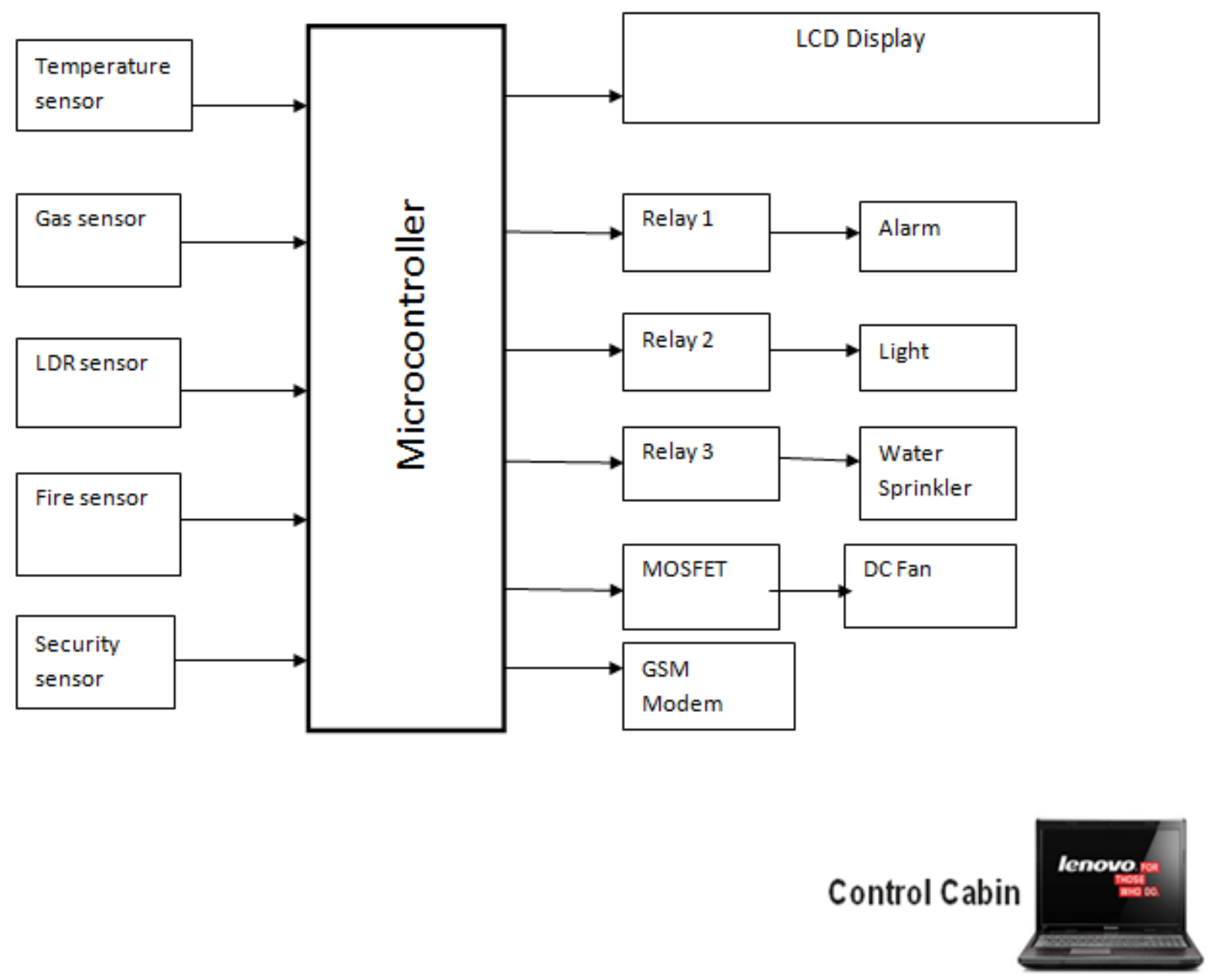

Fig 2

\section{CONCLUSION}

Wireless Sensor Networks have the broad area of applications. This technology can be used in such environments where humans cannot go or it is dangerous for their life like fire. Hence the survey on WSN shows that how we can use WSN for fire detection, which reduce the loss done by fire and we can overcome from it at early stage. The sensor node system is developing for fire detection.

\section{REFERENCES}

[1] Fire Accident Detection and Prevention monitoring System using Wireless Sensor Network enabled Android Application M. Samarasimha Reddy and K. Raghava Rao Indian Journal of Science and Technology, May 2016

[2] Forest Monitoring and Wild land Early Fire Detection by a Hierarchical Wireless Sensor Network by Molina-Pico,David Cuesta-Frau, Alvaro Araujo, Javier Alejandre, and Alba Rozas Hindawi publishing corporations 2016
[3] Energy Efficient Wireless Sensor Network For Polyhouse Monitoring Suprotim Sinha Majumdar, Marut Pattanaik and JV Alamelu European Journal of Advances in Engineering and Technology, 2015

[4] Cracker industry Fire monitoring System Over cluster based WSN By-K.Aanandha Saravanan, N.VigeshPrasanna et.al. Journei of Engg. And applied science 2014

[5] Wireless Sensor Network for Oil \& Gas Industry Tallita Sobral Silicon reef2012

[6] Wireless Sensor Networks for Fire Emergency and Gas Detection Amro Qandour, Daryoush Habibi and Iftekhar Ahmad Centre for Communication Engineering Research (CCER) 2012

[7] Wireless sensor network-based fire detection, alarming, monitoring and prevention system for Bord-and-Pillar coal mines Sudipta Bhattacharjee Pramit Roy Soumalya Ghosh Sudip Misra Mohammad S.Obaidat 2012

[8] Wireless Sensor Network Applications: A Study in Environment Monitoring System Mohd Fauzi Othman, Khairunnisa Shazali International S ymposium on R obotics and Intelligent Sensors 2012 
[9] Wireless Sensor Network based Fire Monitoring and Extinguishing System in Real Time Environment P. N. Narendra Reddy, P. I. Basarkod, S. S. Manvi Int. J. Advanced Networking and Applications 2011

[10] Long distance wireless sensor networks applied in coal mine Yuan, Zhang Shen Wang Quan-fu Song Pei The $6^{\text {th }}$ International Conference on Mining Science \& Technology 2009

[11] Design and Implementation of Automatic Fire Alarm System based on Wireless Sensor Networks Lei Zhang, and Gaofeng Wang International Symposium on Information Processing 2009

[12] A Design and Implementation of Forest-Fires Surveillance System based on Wireless Sensor Networks for South Korea Mountains Byungrak Son, Yong sork Her, and Jung Gyu Kim JCSNS International Journal of Computer Science and Network Security 2006 